\title{
Wrapping the pancreas with a polyglycolic acid sheet be- fore stapling reduces the risk of fluid collection on the pancreatic stump after distal pancreatectomy
}

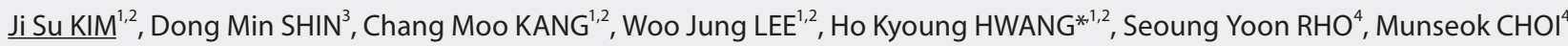 \\ 'Department of Hepatobiliary and Pancreatic Surgery, Yonsei University College of Medicine, Seoul, Korea \\ ${ }^{2}$ Pancreatobiliary Cancer Center, Yonsei Cancer Center, Severance Hospital, Seoul, Korea \\ ${ }^{3}$ Department of Surgery, Yonsei University College of Medicine, Seoul, Korea \\ ${ }^{4}$ Department of Surgery, Yongin Severance Hospital, Yongin, Korea
}

Introduction: Postoperative pancreatic fistula (POPF) and postoperative fluid collection (POFC) are common complications after distal pancreatectomy (DP). The previous method of reducing the risk of POPF was the application of a polyglycolic acid (PGA) sheet to the pancreatic stump after cutting the pancreas with a stapler (After-stapling); the new method involves wrapping the pancreatic resection line with a PGA sheet before stapling (Before-stapling). The study aimed to compare the incidence of POPF and POFC between two methods.

Methods: Data of patients who underwent open or laparoscopic DPs by a single surgeon from October 2010 to February 2020 in a tertiary referral hospital were retrospectively analyzed. POPF was defined according the updated International Study Group of Pancreatic Fistula criteria. POFC was measured by postoperative computed tomography (CT).

Results: Altogether, 182 patients were enrolled (After-stapling group, $\mathrm{n}=138$; Before-stapling group, $\mathrm{n}=44$ ). Clinicopathologic and intraoperative findings between the two groups were similar. Clinically relevant POPF rates were similar between both groups (4.3\% vs. $4.5 \%, p=0.989)$. POFC was significantly lesser in the Before-stapling group on postoperative day $7(p<0.001)$.

Conclusions: Wrapping the pancreas with PGA sheet before stapling was a simple and effective way to reduce POFC. 